\title{
Progenitor cell-derived basophils: a novel barcoded passive degranulation assay in allergy diagnosis
}

\author{
Jiakai $\mathrm{Wu}^{1}$, Rajia Bahri ${ }^{1}$, Marina Tsoumani ${ }^{2}$, Aida Semic-Jusufagic ${ }^{1}$, Clare Murray ${ }^{1}$, A \\ Custovic $^{3}$, George Guibas ${ }^{1}$, Miriam Bennett ${ }^{1}$, Gail Gauvreau ${ }^{4}$, Ruth Cusack ${ }^{4}$, Clare Mills ${ }^{1}$, \\ Silvia Bulfone-Paus ${ }^{1}$, and Angela Simpson ${ }^{1}$ \\ ${ }^{1}$ The University of Manchester \\ ${ }^{2}$ University of Manchester \\ ${ }^{3}$ Imperial College London \\ ${ }^{4}$ McMaster University
}

June 22, 2020

\begin{abstract}
Background: Effector cells assays provide an overall measure of responsiveness to allergen, but the lack of reliable, highthroughput assays limits the clinical utility of this approach. The aim of this study was to develop a high-throughput Basophil Activation Test (BAT), based on human progenitor cell-derived basophils (PCB), and to investigate the role of PCB activation test (PCBAT) in allergy diagnosis. Methods: PCBs were differentiated from CD34+ progenitor cells, and sensitized with sera from subjects sensitized to cat $(\mathrm{n}=35,17$ subjects clinical reactivity validated), peanut-allergic $(\mathrm{n}=30,15$ subjects clinical reactivity validated), peanut-sensitized but tolerant subjects $(n=13)$. Sensitized PCBs were then stimulated with a range of concentrations of the corresponding allergens and degranulation was measured using CD63 expression on flow cytometry. Results: Following passive sensitisation of the mature PCB (2D7+/FceRI+/CD117-/HLADR-) with serum and stimulation with allergen, we saw a dose-dependent increase in CD63 expression which was allergen specific. In subjects sensititsed to cat there was a positive correlation between PCBAT area under curve (AUC) versus specific IgE ( $\operatorname{sgE}$ ) to cat ( $\mathrm{p}=0.001)$ and versus airway responsiveness to inhaled cat allergen $(\mathrm{p}=0.026)$. There was a significant negative correlation between PCBAT AUC for peanut allergen and response to oral food challenge test to peanut - subjects with higher PCBAT AUC reacted to a lower dose on the oral food challenge to peanut $(\mathrm{p}=0.001)$, and had higher sIgE to Ara $\mathrm{h} 1(\mathrm{p}=0.007)$. All peanut tolerant subjects showed no reaction to peanut on PCBAT. Conclusion: PCBAT may confer a powerful alternative tool in allergy testing.
\end{abstract}

\section{Keywords: basophil, allergy diagnosis, challenge tests, food allergy, asthma, flow cytometry}

\section{Introduction}

Sensitisation to inhalant allergens such as house dust mites, cats and dogs, is commonly associated with asthma, but is neither necessary nor sufficient for disease expression. Similarly, a positive skin prick test (SPT) or IgE test to a food does not equate to clinical food allergy, and false positive results are common. Challenge testing (either oral food challenge or inhaled allergen challenge) can be offered to patients, but is time consuming, carries the risk of severe reaction and is not suitable for all patients. Therefore, tests with superior diagnostic accuracy than IgE that are safe to conduct in all patients would be of value in clinical practice, especially amongst patients sensitised to many allergens. 
Basophils and mast cells (MC) are the two primary effector cells in allergic responses (1). Cellular degranulation triggers the release of preformed and newly synthesized mediators inducing a potent biological response in a sensitized person following allergen exposure (2). While basophils are found in the circulation, MC are localized in peripheral tissues. The two cell types may have different roles in an allergic response but this is currently poorly understood $(2,3)$.

The more accessible circulating basophils have been used as cell models for studying allergy (4). Basophils account for $<1 \%$ of blood leukocytes however, making purification a challenge. To obviate the need for purification, the basophil activation test (BAT) was developed using immediately analysed fresh whole blood $(4,5)$. Following stimulation of whole blood with allergen (or control), the responsiveness of the basophils can be quantified using fluorochrome-coupled antibody markers of basophil activation (e.g. CD63 and CD203c) by flow cytometry. The advantage of the BAT is that it takes account of many factors which influence basophil responsiveness to an allergen such as $\operatorname{IgG} 4 / \operatorname{IgE}$ ratio $(6,7)$, heterogeneity of $\operatorname{sIgE}$ to allergen components (8), medication (9) and innate responsiveness of the cells (10). The disadvantages are that blood needs to be analysed immediately after being drawn (5), requiring the allergy clinic to have instant access to a staffed flow cytometry facility. In addition, 10-20\% of people carry "non-releaser" basophils, which are non-responsive in the BAT, despite having clinical allergy (11). Consequently, this test is not generally available for clinical diagnostics, but used only in specialist laboratories for hymenoptera venom and drug allergy testing.

The passive BAT, which uses basophils from a donor that are passively sensitized with the serum from the patient, was developed as an alternative method that circumvents some of these problems (12). Stored serum samples from subjects can be analysed in batches, providing greater flexibility and allowing humoral factors to be investigated separately from cellular factors (7). However, the donors' basophils must be stripped of endogenous IgE with a mild acid treatment before the cells can be passively sensitized with patient serum samples, which can damage the donor basophils and lead to auto-basophil activation (13) and reduced sensitivity (14). Due to these limitations, passive BAT has only been used in a few studies. Although the passively sensitized approach has also been used on basophilic cell lines such as RBL-2H3, there are a number of disadvantages, including the gradual loss of cell responsiveness within weeks of cultures (15).

Recently, Bahri et al developed a robust and reproducible effector cell assay based on human progenitor cell derived MC, the mast cell activation test (MAT). This assay appeared to confer superior diagnostic accuracy in distinguishing peanut allergic from peanut tolerant subjects compared with existing diagnostics such as SIgE (to whole peanut or Ara h 2), SPT and BAT (16). In this study we use a similar approach to generate functional progenitor cell-derived basophils $(\mathrm{PCB})$ and provide detailed characterization of basophil differentiation, and demonstrate the functionality and reproducibility of this technique. We then explore the potential clinical application of progenitor cell basophil activation test (PCBAT) by passively sensitizing the cells with sera/plasma from five groups of patients with allergic asthma and food allergy and testing degranulation to two allergens (cat and peanut).

\section{Methods}

\section{Study design.}

We developed a new high-throughput Basophil Activation Test, using basophils generated from peripheral blood progenitor cells from healthy donors - the PCBAT. To assess the potential clinical utility, serum samples from study participants were used to passively sensitise the basophils, which were then incubated with allergen before assessing basophil activation using flow cytometry. The association between the degree of basophil activation and clinical characteristics of the study participants was then assessed. 


\section{Materials}

\section{Development of progenitor cells-derived basophil activation test (PCBAT)}

\section{Generating PCBs}

Peripheral blood mononuclear cells (PBMCs) were isolated from leukocyte cones (NHS Blood and Transplant Centre, Manchester) using Ficoll density gradient centrifugation. CD34 ${ }^{+}$hematopoietic progenitor cells were isolated by a magnetic bead method according to manufacturer's instructions (MACS Miltenyl Biotec). Purified CD34 ${ }^{+}$hematopoietic progenitor cells were diluted to $1 \times 10^{5}$ cells $/ \mathrm{ml}$ and cultured in Stemspan $^{\text {TM }}$ supplemented with $10 \mathrm{ng} / \mathrm{ml}$ IL-3, $100 \mathrm{ng} / \mathrm{ml} \mathrm{SCF}, 50 \mathrm{ng} / \mathrm{ml} \mathrm{IL-6,} \mathrm{5mg/ml} \mathrm{human} \mathrm{LDL} \mathrm{and}$ penicillin/streptomycin $(100 \mathrm{U} / \mathrm{ml})$. This was day 0 of culture, cell density was then maintained between $2-5$ x $10^{5} / \mathrm{ml}$ up to day 28 at $37^{\circ} \mathrm{C}$ with $5 \% \mathrm{CO}_{2}$.

\section{PCBs characterization}

To monitor the differentiation process, the culture was sampled at day $7,10,16,21$ and 28 . The cells were characterized using flow cytometry, immunofluorescence and metachromatic staining and by functional assay (PCBAT). This was repeated on two separate donors.

\section{Flow cytometry}

Cell staining was performed on a 96-well plate using approximately $5 \times 10^{4}$ cells/well. For PCB characterization, cells were stained with the following antibodies: CD63 (APC), CD123 (Percp-Cy5.5), CD117 (BV605), CD203c (FITC), HLADR (eFluro450) and FceRI (PE-Cy7) for 20 minutes at $4^{\circ} \mathrm{C}$. Detailed protocol and gating strategy can be found in Figure E1-2.

\section{Immunofluorescence staining}

Cells were fixed in $4 \%$ paraformaldehyde and permeabilized with $0.1 \%$ tween and $10 \%$ goat serum. Cells were then stained in mouse anti-BB1 antibody (1:10) followed by Alexa Fluor 555 goat anti-mouse secondary antibody (1:200). Slides were mounted with fluoroshield mountant containing DAPI for cell nuclear staining and examined under a Leica DM IL LED microscope using Leica Application Suite software (Leica, UK).

\section{Validation and performance of PCBAT}

\section{Study subjects}

Five groups with different clinical characteristics were identified and described in Table 1. All subjects provided written informed consent.

\section{Measurement of sensitisation to cat and peanut allergens}

Serum sIgE was measured for Groups 1 and 2 to cat and for Group 4 to peanut and peanut components allergens Ara h 1, 2, 3, 8, 9 (Immunocap, ThermoFisher Scientifc, Sweden); sIgE $>0.35 \mathrm{kU} / \mathrm{L}$ indicated a positive test. For Group 5, serum sIgE to Ara h 1, 2, 3, 6, 8, 9 was measured using ISAC (ThermoFisher Scientifc, Sweden). A positive result was indicated by sIgE $>0.3$ ISU-E. Serum sIgE to cat was not available for Group 3 and sensitisation was determined by titrated cat allergen SPT (17).

\section{Inhaled cat allergen challenge}

Seventeen cat sensitized adults underwent inhaled cat allergen challenge at McMaster University, Ontario. Participants inhaled cat allergen at increasing concentrations until lung function $\left(\mathrm{FEV}_{1}\right)$ dropped $>20 \%$ from baseline. To quantify airway responsiveness to cat allergen, we calculated a dose response slope (DRS) and also the $\mathrm{PC}_{20}$ to cat allergen (described in this article's Online Repository) (18). FEV 1 was measured 
for 7 hours after the last dose of inhaled allergen and recovery was measured as AUC; the early asthmatic response AUC between 0-2 hours post challenge (EARAUC 0 -2hrs) and the late asthmatic response AUC between 3-7 hours post challenge (LARAUC $3-7 \mathrm{hrs}$ ) (described in this article's Online Repository).

\section{Oral peanut challenge}

Of thirty physician confirmed peanut allergic patients, 15 underwent oral food challenge to peanut as part of iFAAM project (19), (challenge protocol in the article's Online Repository). Participants ingested increasing quantities of peanut protein until objective signs of an allergic reaction were shown. The cumulative dose of peanut required to show first objective sign was used as a measure of clinical reactivity to peanut allergen.

\section{PCBAT}

PCBs were sensitized with either $20 \%$ patients' sera or with human myeloma $\operatorname{IgE}(1 \mu \mathrm{g} / \mathrm{ml})$ overnight at $37^{\circ} \mathrm{C}$ with $5 \% \mathrm{CO}_{2}$. Sensitized PCBs were activated by incubating with serial dilutions of extracts (roasted peanut extracts or cat allergen) for 30 minutes at $37^{\circ} \mathrm{C}$. Anti- $\mathrm{IgE}(1 \mu \mathrm{g} / \mathrm{ml})$ and "medium only" was included for every subject as positive and negative controls respectively. PCBs were identified by staining the

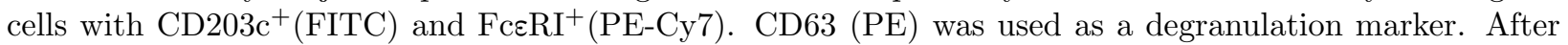
cells were stained with antibodies and viability dyes, Fluorescent barcoding (16-plex) was performed using methods previously described (20). Briefly, cells were fixed with $1.6 \%$ formaldehyde then permeabilized with methanol containing pacific blue $(40,13.3,4.43$ and $0 \mu \mathrm{g} / \mathrm{ml})$ and Alexafluro $700(4,1.33,0.43$ and $0 \mu \mathrm{g} / \mathrm{ml})$. Cells stained with different combinations of pacific blue and Alexafluro 700 were then pooled before flow cytometry analysis. A minimum 5\% of CD63 positive cells were required to indicate a positive PCBAT response. To depict the responsiveness of the PCBAT, we present results as AUC for CD63 expression at increasing allergen concentrations; results for sensitivity (EC50 and CDsens) and reactivity (CDmax) of the PCBAT are presented in Table E1-E5.

\section{Statistics}

Demographic variables were presented as means and standard deviation. The AUC was calculated using the trapezoidal rule on logarithmically transformed allergen concentrations to quantify the responsiveness of a degranulation assay (21), as previously described. Methods for EC50, CDsens and CDmax calculation is described in this articles' Online Repository. Correlation coefficients were calculated by using the Spearman $\mathrm{R}$ test in (SPSS v22, IBM, Armonk, USA). A 2-sided P value [?] 0.05 was considered statistically significant.

\section{Results}

\section{PCBAT Development}

\section{Characterization of PCBs maturity}

Human primary PCBs were differentiated from CD34 ${ }^{+}$hematopoietic progenitors following 28 days of culture. The maturity of PCBs was demonstrated using four methods - immunophenotyping (Figure1 A-C), immunofluorescence staining (Figure 1D), functional tests (Figure 1E) and morphological study (Figure E3) for PCBs obtained from 2 different donors (donor A in Figure 1 and donor B in Figure E4).

Mature PCBs were defined as $2 \mathrm{D} 7^{+} / \mathrm{FcERI}^{+} / \mathrm{CD} 117^{-} / \mathrm{HLADR}^{-}$cells. The proportion of cells expressing the basophil marker 2D7 increased from day $7(32.8 \%)$ peaked at day $16(42.8 \%)$ and fell to $22.1 \%$ by day 28 (Figure 1A). When the $2 \mathrm{D}^{+}$cells were gated for $\mathrm{FcERI}^{+}$, the majority were positive, around $80 \%$ of the $2 \mathrm{D} 7^{+}$cells were also $\mathrm{FcERI}^{+}$through the culture (Figure 1B). Furthermore, as the cells matured, the highest negativity for $\mathrm{CD}_{117^{-}}$and $\mathrm{HLADR}^{-}$was seen at day 16 (Figure 1C). However, by day 28, the cells 
started to gain CD117 receptor expression, an additional indication that the culture was losing basophilic characteristics.

We performed immunofluorescence staining with another basophil marker, BB1. The BB1 positive cells were faintly visible at day 7 , but clearly visible by day 16 and remained visible to day 28 (Figure 1D).

We tested the PCBs' ability to degranulate upon engagement of the FcєRI by sensitising them with recombinant IgE followed by anti-IgE stimulation. The percentage of basophil activity, as measured by CD63 expression, increased as the cells matured, such that by day 16, 36.5\% of cells showed degranulation (Figure $1 \mathrm{E})$. This was repeated with PCBs from 4 other donors and showed a consistent percentage of activation between donors at day 16 (mean \pm SEM $41.31 \% \pm 3.34$, Figure E5A and E5B). The maturation of PCBs and degranulation capability at each stage was very similar between the two donors (Figure 1, E4 and E5C).

Using this culture method, an average yield $(\mathrm{n}=9)$ of $2.27 \times 10^{7}$ cells $\left(9 \times 10^{6}-4.8 \times 10^{7}\right.$, minimum-maximum) could be achieved at day 16 of culture of which 25-50\% were basophils (Figure E6). May-Grünwald Giemsa staining performed on day 16 cells showed heavily granulated cells (Figure E3), a morphology resembling that of blood basophils previously reported (22).

The combined use of immunophenotyping, immunofluorescence, morphological characterization and functional tests suggested that the optimal window for PCBs was between day 16 and day 21. After day 21, cells began to lose basophilic features but still retained a high response to anti-IgE following IgE sensitization (Figures $1 \mathrm{~A}$ and $1 \mathrm{E})$.

\section{High-throughput PCBAT with fluorescent barcoding}

To increase the throughput, we incorporated fluorescent barcoding to the PCBAT. We simplified the gating strategies for selecting $\mathrm{PCB}$ population to minimize the interference between fluorescence dyes and the antibody panel. We selected $\mathrm{CD} 203 \mathrm{c}^{+/} \mathrm{FcERI}^{+}$cells for the degranulation assay, as CD203c ${ }^{+}$cells were $>99 \% 2 \mathrm{D}^{+}$(Figure E6). In addition, amongst CD203c ${ }^{+}$cells, only FceRI ${ }^{+}$population could degranulate in response to FceRI crosslinking (Figure E7). In Figure 2 we showed a representative figure for the use of 16-plex fluorescence barcoding in PCBAT.

\section{PCBAT predicts clinical reactivity to cat}

Study groups and controls are described in Table I with individual subject PCBAT results and available skin test results are presented in Table E1-E4. All the samples responded to positive control stimulant (anti-IgE) and did not respond to negative control stimulant (medium only).

For adults with asthma sensitised to cat (Group 1, $\mathrm{n}=18$ ), all but one subject showed a positive response in PCBAT (Figure 3A) with a broad range of trajectories and AUC. There was a significant correlation between AUC in PCBAT and sIgE to cat (Figure 3B p=0.001). Five of the six control subjects with asthma who were not sensitised to cat showed a negative response to cat (Figure 3C); one control subject (control 6) showed a weak positive response at the highest concentration.

Four asthma patients were undergoing treatment with the anti-IgE drug omalizumab, three were cat sensitized (Group 2, Figure 3D). No response was seen to cat extract amongst cat-sensitised asthma patients on omalizumab.

For 17 adults with airway reactivity to cat (Group 3) the range of cumulative dose of inhaled cat allergen required to cause a [?]20\% drop in $\mathrm{FEV}_{1}$ was large $\left(>2000\right.$-fold, Figure E8A), as were the DRS, and $\mathrm{PC}_{20}$ (Figure E8B-C). The DRS and $\mathrm{PC}_{20}$ were closely correlated (Figure $\mathrm{E} 8 \mathrm{D}, \mathrm{p}=0.0001 \mathrm{R}^{2}=0.94$ ). Although specific IgE to cat was not available on these subjects, titrated skin test reactivity to cat showed a range of sensitivity to cat (7 doubling dilutions, Figure E8E). Fifteen subjects showed a positive response on PCBAT, with a broad range of trajectories (Figure 4A). There was no association between skin test reactivity to cat and DRS to cat allergen (Figure E8F, p=0.17). There was a significant correlation between PCBAT AUC 
and airway reactivity DRS (Figure $4 \mathrm{~B}, \mathrm{p}=0.026$ ) and the $\operatorname{lnEARAUC}_{0-2 \mathrm{hrs}}$ after inhaled allergen challenge (Figure $\mathrm{E} 8 \mathrm{G}, \mathrm{p}=0.038$ ) but not $\operatorname{lnLARAUC}$-7hrs $($ Figure $\mathrm{E} 8 \mathrm{H}, \mathrm{p}=0.29)$. All control subjects $(\mathrm{n}=6)$ showed no response in PCBAT (Figure 4C).

\section{PCBAT predicts clinical reactivity to peanut}

For adults with physician diagnosed peanut allergy $(\mathrm{n}=30$, Group 4) all subjects showed a positive response on PCBAT which was dose-dependent (Figure 5A). The four negative control subjects did not respond to peanut on PCBAT (Figure 5B). There was a significant correlation between AUC in PCBAT and SIgE to whole peanut and Ara h 1, 2 (Figure 5C-E), 3 and 6 (Figure E9A and D). Serum sIgE to Ara h 8 and 9 were not significantly associated with AUC (Figure E9B and C, p>0.7).

Of the 30 subjects with physician diagnosed peanut allergy (Group 4), 15 had confirmed peanut allergy following double blind placebo control food challenge. There was a significant negative correlation between PCBAT AUC and results of oral food challenge test to peanut - subjects who showed a higher PCBAT AUC reacted to a lower dose on oral food challenge to peanut (Figure $5 \mathrm{~F} \mathrm{p}=0.001, \mathrm{R}^{2}=0.57$ ). A trend towards a negative correlation was observed between sIgE to whole peanut and oral food challenge result (Figure 5G, $\mathrm{p}=0.094, \mathrm{R}^{2}=0.24$ ). A significant negative correlation was also observed between $\operatorname{sgE}$ to Ara $\mathrm{h} 1$ and oral food challenge result (Figure $5 \mathrm{H} \mathrm{p}=0.007, \mathrm{R}^{2}=0.55$ ). There was no significant association between Ara $\mathrm{h} 2$ $\mathrm{sIgE}$ and oral food challenge result (Figure 5I, $\mathrm{p}=0.125, \mathrm{R}^{2}=0.2$ ).

Within a population-based birth cohort (Group 5), we identified 13 subjects who were sensitised to peanut (positive to 1 or more allergen component; 5 sensitised to Ara h 1, 2, 3 or 6, Table E6) who reported regular ingestion of peanut (i.e. sensitized but tolerant to peanut, Group 6). None of these 13 subjects showed responsiveness to even the highest concentration of peanut extracts in PCBAT (Figure 5J).

\section{Discussion}

We have developed a high-throughput progenitor cell derived basophil activation test (PCBAT), which was a better predictor of clinical reactivity to cat and peanut allergen (as measured on challenge testing) than conventional markers of allergy such as SPT or allergen specific IgE. PCBAT can be used with stored serum, removing the need for immediate access to expensive flow cytometry facilities not generally available in the clinic. By passively sensitising basophils with sera from our well characterised patient populations then culturing with the relevant allergen, we demonstrated dose dependent and allergen specific basophil activation with wide variability in trajectories. Importantly, PCBAT was negative amongst those reporting oral tolerance to peanut but with detectable specific $\operatorname{IgE}$ to peanut components.

\section{Technical aspects of the PCBAT}

The combined use of flow cytometry and immunostaining suggest the optimal window for maturation for this culture protocol was between day 16 and 21, similar to previous reports. (23), and reflecting the 5 days basophil lifespan in vivo (24). Our culture was highly enriched for mature basophils (25-50\%), enabling high-throughput barcoding, improving efficiency.

\section{$\mathrm{PCB}$ responsiveness and $\mathrm{sIgE}$ levels}

The responsiveness of PCBAT was allergen specific showing a dose-dependent response with good association with levels of corresponding SIgE. In addition, patients receiving omalizumab treatment showed completely muted responsiveness in the PCBAT, in accordance with a previous study (25).

However, one subject, with low but positive sIgE to cat $(0.5 \mathrm{KU} / \mathrm{L})$ did not show responsiveness in PCBAT; this patient was not on omalizumab treatment. As this subject had not undergone allergen challenge, it 
remains unclear whether this subject was sensitized but tolerant to cat. One non-sensitized control subject showed minor degranulation at the highest concentration of cat allergen used. We have confirmed that this subject had a positive $\operatorname{dog} \operatorname{sIgE}(5 \mathrm{KU} / \mathrm{L})$; we speculate that this weak response to cat allergen might reflect cross-reactivity between cat allergen and $\operatorname{dog} \operatorname{sIgE}$, which has been previously reported (26-28). We also found quantifiable traces of Can $\mathrm{f} 1$ in the cat allergen extracts used which may provide an alternative explanation (Figure E10).

\section{The clinical relevance of PCBAT}

We investigated the clinical relevance of PCBAT using two cohorts of patients who had undergone challenge testing - to inhaled cat allergen or to peanuts.

Although a significant association was observed between PCBAT AUC and inhalant challenge results, two subjects showed negative results in the PCBAT but positive in the inhalant challenge. Interestingly, these two subjects exhibited the smallest reaction in the skin test to cat at the time of challenge $(2 \times 2 \mathrm{~mm})$ and had significantly greater reactivity to other allergen including dust mite and grass (Table E4). In addition, for some of these subjects, the blood samples were collected up to 2 years after the inhaled allergen challenge, so a change in clinical reactivity during this time remains a possible explanation

We explored PCBAT in peanut allergy, as an exemplar of an allergic disease where clinical reactivity is not reliably predicted by serum SIgE, but where oral food challenge tests are used to confirm reactivity and identify thresholds of responsiveness. All 30 patients with physician diagnosed peanut allergy showed a positive PCBAT, and this correlated significantly with serum sIgE to whole peanut extract and to Ara $h$ 1,2 and 3. As half of the subjects had previously undergone oral food challenge to peanut, we were able to compare the dose of peanut at which the subject showed an objective reaction with the reactivity on the PCBAT (as AUC). We identified that AUC on PCBAT was a better predictor of clinical reactivity on oral food challenge than serum sIgE to whole peanut extract or to Ara h 2 and 3, and was similar to Ara $h 1$. Importantly we identified a small population with positive sIgE to 1 or more peanut allergen component that reported regularly eating peanuts, and found that none of them showed a positive PCBAT. This suggests that in principle, PCBAT may be a useful test in predicting clinical reactivity to peanut, but further testing on more subjects would be required.

\section{Conclusion}

By generating progenitor-cell derived basophils in high-yield we have developed a flow cytometry-based basophil activation test for use with stored serum which can be used to assess reactivity to both food and inhalant allergens. By incorporating fluorescent barcoding, we have increased the throughput of the assay, reducing costs significantly. We identified wide variability in trajectories of response to allergen in different subjects, and responses were muted in the presence of the anti-IgE treatment omalizumab, indicating that this test better reflects the overall immune milieu rather than just specific IgE. For subjects who had undergone oral food challenge to peanut, we were able to use results of PCBAT to predict clinical reactivity to peanut. The correlation of PCBAT to clinical reactivity to inhaled cat allergen may represent a safe and robust way of identifying those asthmatics who might benefit from interventions for ongoing cat allergen exposure. Although further evaluation is required, this proof of concept study indicates that this test may have a role in food and inhalant allergy testing as a means of identifying clinically important sensitisations.

Table I Demographic description of the study groups.

\begin{tabular}{lll}
\hline Group number & allergen used in PCBAT & Study group description \\
1 & Cat & Sensitised to cat
\end{tabular}




\begin{tabular}{lll}
\hline 2 & Cat & Asthma, on Omalizumab, sensitised to inhalant allergens \\
3 & Cat & Clinical reactivity quantified with inhaled allergen challenge to cat \\
4 & Peanut & Doctor diagnosed peanut allergy (clinical reactivity quantified using oral food \\
5 & Peanut & Sensitisation to 1 or more peanut allergen components, but self-reported ingest \\
\hline
\end{tabular}

\author{
* Patients recruited from ManARTS biobank (Rec reference: 15/NW/0409) \\ ** From McMaster University, Ontario, Canada \\ *** From the population birth cohort-Manchester Asthma and Allergy Study (MAAS, registration: \\ ICRCTN72673620) detailed elsewhere (29)
}

\title{
References
}

1. He SH, Zhang HY, Zeng XN, Chen D, Yang PC. Mast cells and basophils are essential for allergies: mechanisms of allergic inflammation and a proposed procedure for diagnosis. Acta pharmacologica Sinica. 2013;34(10):1270-83.

2. Kumar S, Verma AK, Das M, Dwivedi PD. Molecular mechanisms of IgE mediated food allergy. Int Immunopharmacol. 2012;13(4):432-9.

3. Amin K. The role of mast cells in allergic inflammation. Resp Med. 2012;106(1):9-14.

4. MacGlashan DW, Jr. Basophil activation testing. The Journal of allergy and clinical immunology. 2013;132(4):777-87.

5. Santos AF, Lack G. Basophil activation test: food challenge in a test tube or specialist research tool? Clinical and translational allergy. 2016;6:10.

6. Okamoto S, Taniuchi S, Sudo K, Hatano Y, Nakano K, Shimo T, et al. Predictive value of IgE/IgG4 antibody ratio in children with egg allergy. Allergy, asthma, and clinical immunology : official journal of the Canadian Society of Allergy and Clinical Immunology. 2012;8(1):9.

7. Santos AF, James LK, Bahnson HT, Shamji MH, Couto-Francisco NC, Islam S, et al. IgG4 inhibits peanut-induced basophil and mast cell activation in peanut-tolerant children sensitized to peanut major allergens. The Journal of allergy and clinical immunology. 2015;135(5):1249-56.

8. Christensen LH, Holm J, Lund G, Riise E, Lund K. Several distinct properties of the IgE repertoire determine effector cell degranulation in response to allergen challenge. The Journal of allergy and clinical immunology. 2008;122(2):298-304.

9. Hill DA, Siracusa MC, Ruymann KR, Tait Wojno ED, Artis D, Spergel JM. Omalizumab therapy is associated with reduced circulating basophil populations in asthmatic children. Allergy. 2014;69(5):674-7.

10. Youssef LA, Schuyler M, Gilmartin L, Pickett G, Bard JDJ, Tarleton CA, et al. Histamine release from the basophils of control and asthmatic subjects and a comparison of gene expression between "releaser". and "nonreleaser" basophils. Journal of immunology. 2007;178(7):4584-94.

11. Kepley CL, Youssef L, Andrews RP, Wilson BS, Oliver JM. Syk deficiency in nonreleaser basophils. J Allergy Clin Immun. 1999;104(2):279-84.

12. Pruzansky JJ, Grammer LC, Patterson R, Roberts M. Dissociation of IgE from receptors on human basophils. I. Enhanced passive sensitization for histamine release. Journal of immunology. 1983;131(4):194953. 
13. Ishizaka T, Ishizaka K. Mechanisms of passive sensitization. IV. Dissociation of IgE molecules from basophil receptors at acid pH. Journal of immunology. 1974;112(3):1078-84.

14. Moneret-Vautrin DA, Sainte-Laudy J, Kanny G, Fremont S. Human basophil activation measured by CD63 expression and LTC4 release in IgE-mediated food allergy. Annals of allergy, asthma \& immunology : official publication of the American College of Allergy, Asthma, \& Immunology. 1999;82(1):33-40.

15. Passante E. Mast cell and basophil cell lines: a compendium. Methods in molecular biology. 2014;1192:101-13.

16. Bahri R, Custovic A, Korosec P, Tsoumani M, Barron M, Wu J, et al. Mast cell activation test in the diagnosis of allergic disease and anaphylaxis. The Journal of allergy and clinical immunology. 2018.

17. Heinzerling L, Mari A, Bergmann KC, Bresciani M, Burbach G, Darsow U, et al. The skin prick test European standards. Clinical and translational allergy. 2013;3(1):3.

18. Cockcroft DW, Davis BE. Methacholine PC20: 1-point formula. Ann Allergy Asthma Immunol. 2007;98(5):498-9.

19. Nitride C, Lee V, Baricevic-Jones I, Adel-Patient K, Baumgartner S, Mills ENC. Integrating Allergen Analysis Within a Risk Assessment Framework: Approaches to Development of Targeted Mass Spectrometry Methods for Allergen Detection and Quantification in the iFAAM Project. Journal of AOAC International. 2018;101(1):83-90.

20. Krutzik PO, Nolan GP. Fluorescent cell barcoding in flow cytometry allows high-throughput drug screening and signaling profiling. Nature methods. 2006;3(5):361-8.

21. Matthews JN, Altman DG, Campbell MJ, Royston P. Analysis of serial measurements in medical research. Bmj. 1990;300(6719):230-5.

22. Lee JJ, McGarry MP. When is a mouse basophil not a basophil? Blood. 2007;109(3):859-61.

23. Kepley CL, Pfeiffer JR, Schwartz LB, Wilson BS, Oliver JM. The identification and characterization of umbilical cord blood-derived human basophils. J Leukoc Biol. 1998;64(4):474-83.

24. Ohnmacht C, Voehringer D. Basophil effector function and homeostasis during helminth infection. Blood. $2009 ; 113(12): 2816-25$.

25. Eckman JA, Sterba PM, Kelly D, Alexander V, Liu MC, Bochner BS, et al. Effects of omalizumab on basophil and mast cell responses using an intranasal cat allergen challenge. The Journal of allergy and clinical immunology. 2010;125(4):889-95 e7.

26. Viander M, Valovirta E, Vanto T, Koivikko A. Cross-reactivity of cat and dog allergen extracts. RAST inhibition studies with special reference to the allergenic activity in saliva and urine. International archives of allergy and applied immunology. 1983;71(3):252-60.

27. Madhurantakam C, Nilsson OB, Uchtenhagen H, Konradsen J, Saarne T, Hogbom E, et al. Crystal structure of the dog lipocalin allergen Can $\mathrm{f} 2$ : implications for cross-reactivity to the cat allergen Fel d 4. Journal of molecular biology. 2010;401(1):68-83.

28. Nilsson OB, Binnmyr J, Zoltowska A, Saarne T, van Hage M, Gronlund H. Characterization of the dog lipocalin allergen Can f 6: the role in cross-reactivity with cat and horse. Allergy. 2012;67(6):751-7.

29. Custovic A, Simpson BM, Murray CS, Lowe L, Woodcock A, Stu NMAA. The National Asthma Campaign Manchester Asthma and Allergy Study. Pediatr Allergy Immu. 2002;13:32-7.

Figure Legends

Figure 1 Characterization of PCBs differentiation during day 7-28 of culture 
(A-C) Representative figures for non-stimulated controls showing expression of basophil selection markers $2 \mathrm{D}^{+} / \mathrm{Fc} \mathrm{RI}^{+} / \mathrm{CD} 117^{-} / \mathrm{HLADR}^{-}$during culture. (D) Parallel analysis of BB1 expression by immunofluorescence staining. (E) Degranulation capability by stimulating IgE sensitized cells with anti-IgE during culture. Gating strategies were the same as illustrated in A-C.

Figure 2 PCBAT with 16-plex fluorescent barcoding

(A) Illustration of 16-plex fluorescent barcoding with Pacific blue and Alexa Fluor 700. (B) A representative figure of a 16-plex fluorescent barcoded sample in a PCBAT.

Figure 3 PCBAT with cat allergen extracts on cat sensitized but clinical reactivity not validated subjects

PCBAT using cat allergen extracts from ALK (dilution factor 1:250; 1:1,250; 1:6,250;1:31,250; 1:156,250 and 1:781,250) were performed on (A) 18 cat sensitized but clinical reactivity not validated subjects; (B) the relationship between the PCBAT AUC from the sensitized groups and the corresponding sIgE level were shown in scatter plot. (C) PCBAT using cat allergen extracts were also performed on 6 atopic but non-cat sensitized subjects and (D) 4 subjects who are sensitized to cat allergen but under omalizumab treatment, spearman test and $\mathrm{R}$ square was calculated and $\mathrm{p}<0.05$ were considered significant.

Figure 4 PCBAT with cat allergen extracts on cat allergic subjects validated with inhalant allergen challenge

PCBAT using cat allergen extracts from HollisterStier (dilution factor 1:250; 1:1,250; 1:6,250; 1:31,250; 1:156,250 and 1:781,250) were performed on (A) 17 clinal reactivity validated cat allergic subjects; (B) Scatter plot showed the relationship between the PCBAT AUC from the allergic groups and the corresponding natural log transformed dosage response slope. (C) PCBAT using cat allergen extracts were also performed on 6 atopic but non-cat sensitized subjects, spearman test and $\mathrm{R}$ square was calculated and $\mathrm{p}<0.05$ were considered significant.

Figure 5 PCBAT with peanut allergen extracts on peanut allergic and peanut tolerant subjects

PCBAT using peanut allergen extracts $(1,10,100$ or $1000 \mathrm{ng} / \mathrm{ml})$ were performed on (A) 30 subjects with physician diagnosed peanut allergy, and (B) on 4 atopic but non-peanut sensitized subjects. The relationship between the PCBAT AUC from the sensitized groups and the corresponding whole peanut sIgE level Ara h 1 sIgE level and Ara h 2 sIgE level were shown in scatter plot (C-E respectively). Fifteen out of 30 sensitized subjects underwent oral food challenge. The relationship between cumulative peanut dose to show first objective sign and PCBAT AUC or sIgE to whole peanut, Ara h 1 and Ara $\mathrm{h} 2$ were shown in F-I respectively. (J) 13 peanut sensitized but tolerant subjects. Anti-IgE was used as positive control stimulant for all tested serum samples. Spearman test and R square was calculated, $\mathrm{p}<0.05$ were considered significant. 


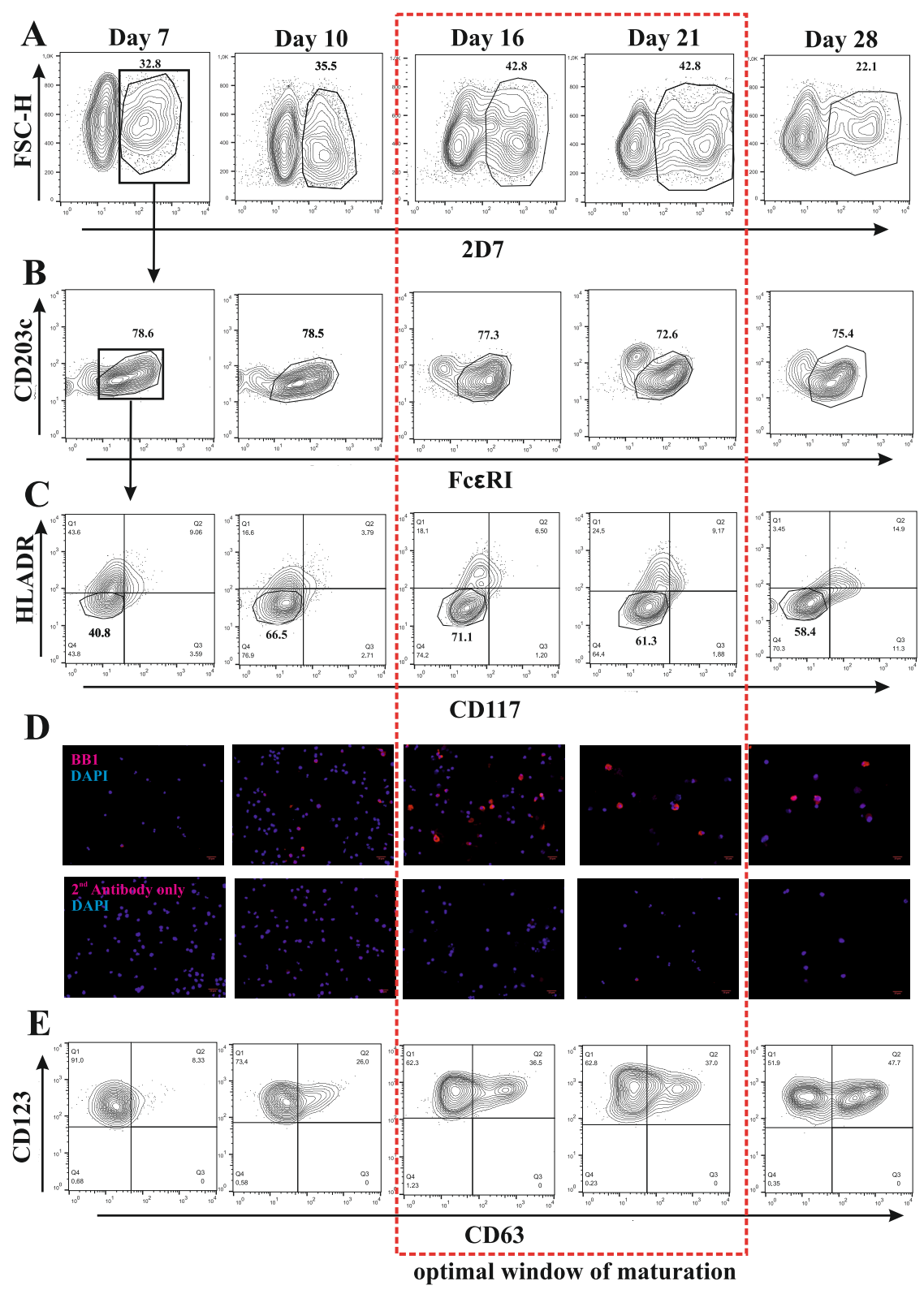




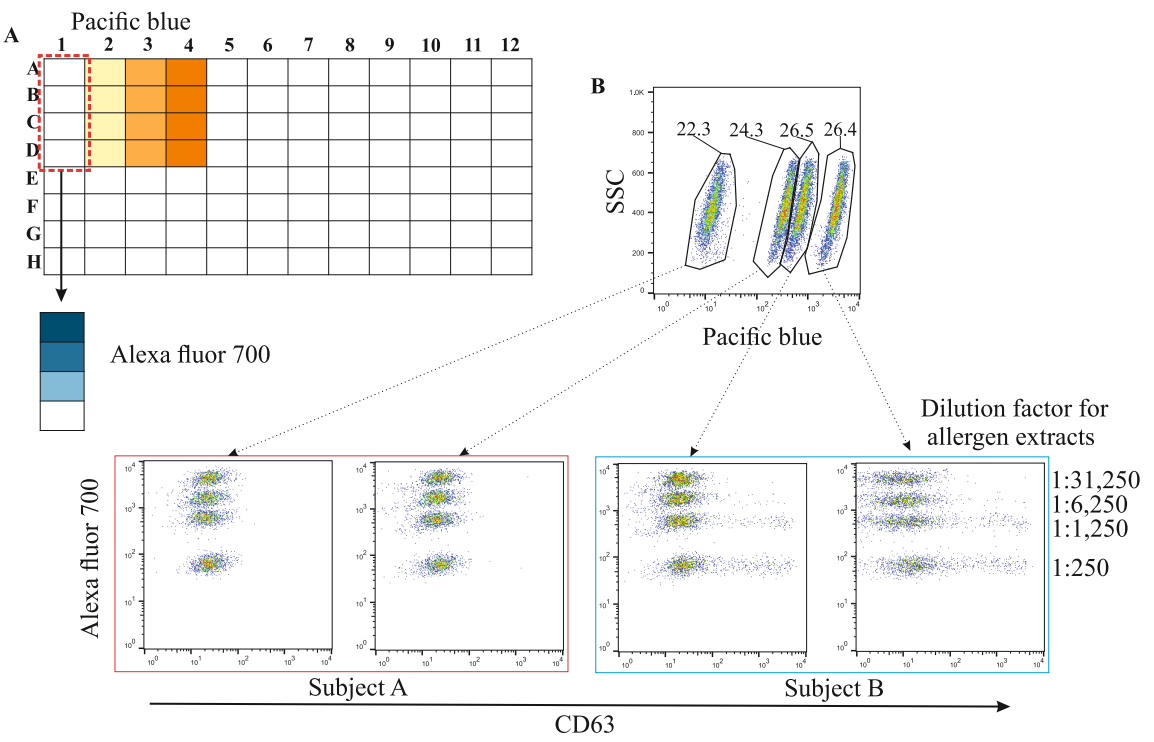

A

B
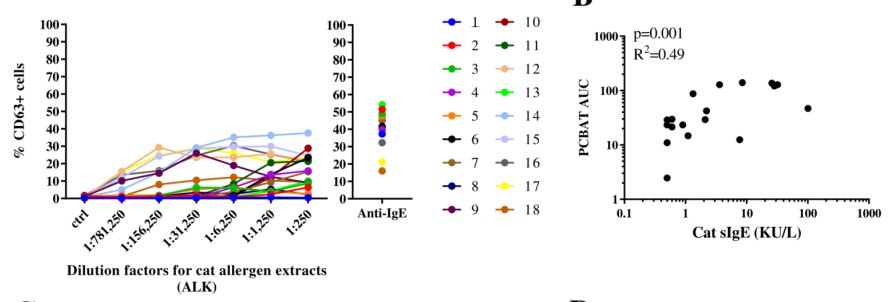

C

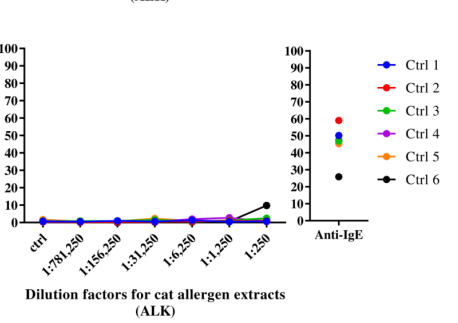

D

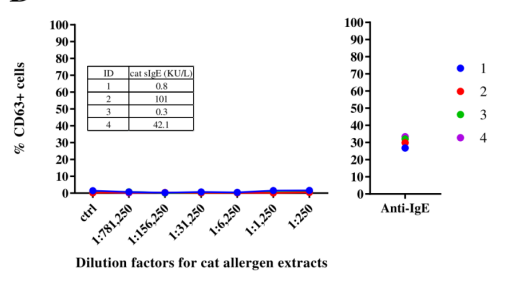


A

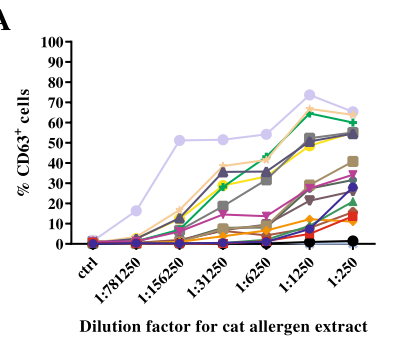

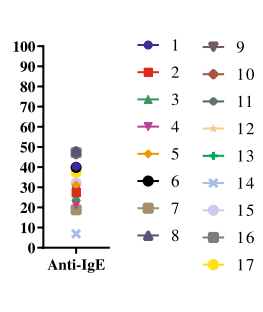

B

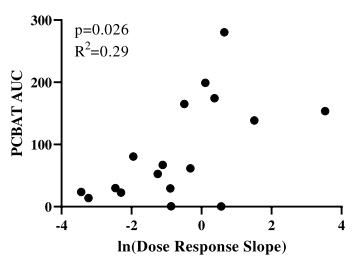

C

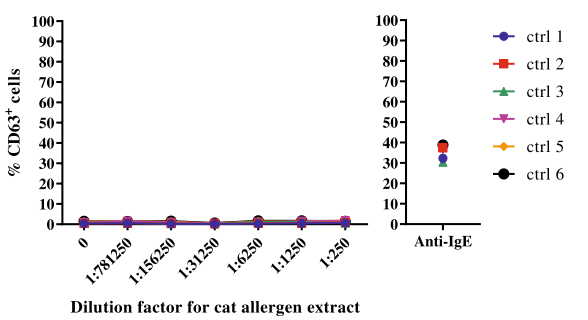

A
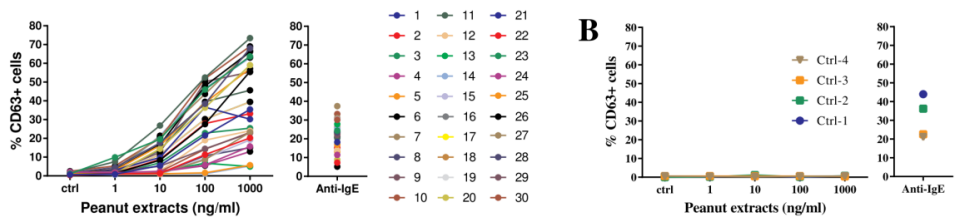

C

D
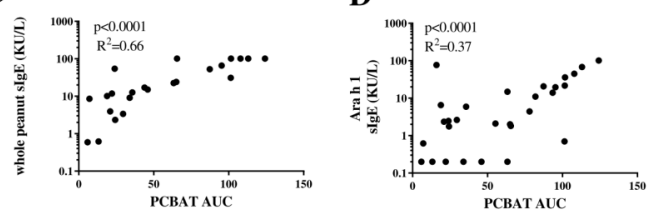

E
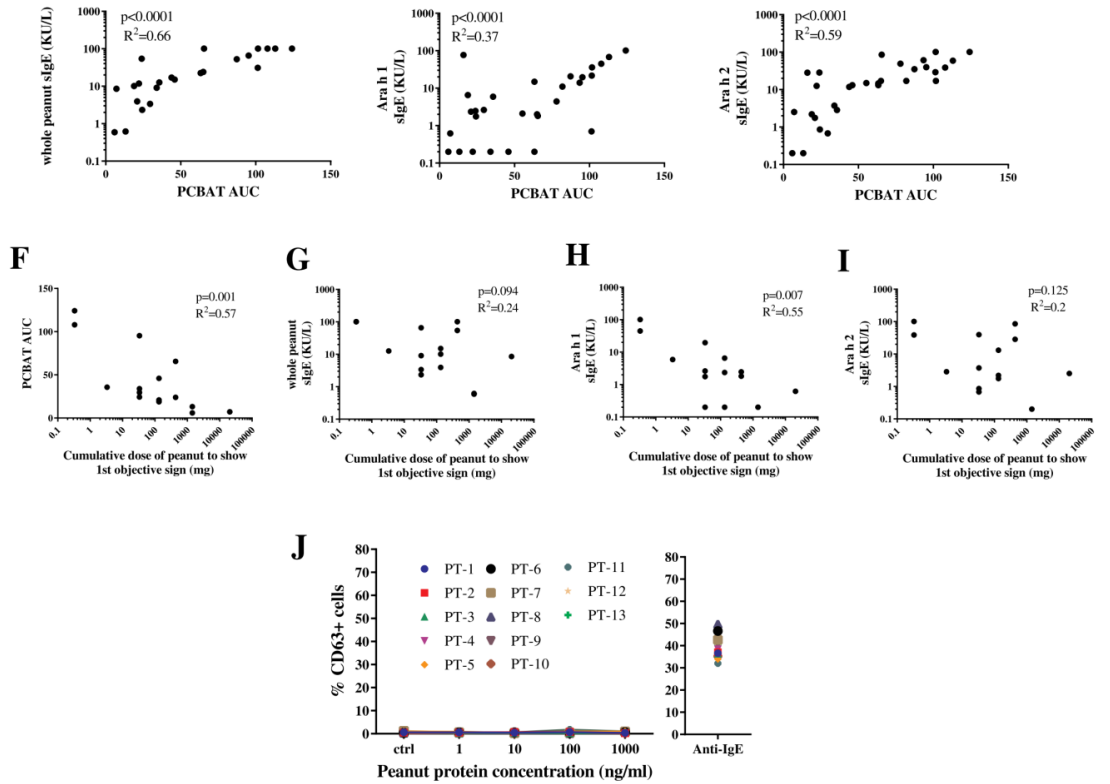